\title{
New Classification for Clinically Symptomatic Adjacent Segment Pathology in Cervical Disc Disease
}

\author{
Srinivasan Sreeramulu Uddanapalli \\ Department of Neurosurgery, MIOT Hospitals, Chennai, India
}

Study Design: Clinical adjacent segment pathology (CASP) is common after cervical disc surgery. A critical examination of 320 patients operated for cervical disc prolapse revealed that CASP can also occur in patients with congenital and degenerative fusion of cervical spine. This has not been studied in depth and there is a need for a practically applicable classification of CASP.

Purpose: To develop a new classification scheme of CASP.

Overview of Literature: A review of the literature did not reveal a practically applicable classification incorporating the occurrence of CASP in congenital and degenerative fusion cases.

Methods: This was a retrospective analysis of 320 patients operated (509 disc spaces) on for cervical disc prolapse. Cases (n=316) were followed-up for 3-11 years. Random sampling of 220 patients with postoperative magnetic resonance imaging (MRI) in 165 cases was analyzed.

Results: Six symptomatic CASP cases required resurgery (1.9\%), eight cases involved MRI proven CASP with axial neck pain only and 13 patients were asymptomatic with radiological adjacent segment pathology (RASP). The frequency rate was 8.5\% (27/316). Four cases of congenital or degenerative fusion of vertebra developed CASP requiring surgery. CASP is classified as primary or secondary follows. Primary A1 was congenital fusion of vertebra and primary A2 was degenerative fusion of the vertebra. Secondary, which was after cervical disc surgery, comprised B1 (RASP in asymptomatic patients), B2 (CASP in patients with axial neck pain), and B3 (CASP with myeloradiculopathy). B3 was subdivided into single-level CASP (B3a) and multiple-level CASP (B3b).

Conclusions: Symptomatic CASP requiring resurgery is infrequent. CASP can occur in patients with congenital and degenerative fusion of the cervical spine. A new classification for CASP along with treatment strategy is proposed. Patients in Primary CASP and B3 CASP require resurgery while others require only observation.

Keywords: Clinical adjacent segment pathology; Adjacent segment degeneration; Adjacent segment disease; Anterior cervical discectomy; Cervical disc prolapse

\section{Introduction}

Adjacent segment degeneration (ASD) is common after cervical disc surgery. ASD has more recently been termed clinical adjacent segment pathology (CASP) [1,2]. The frequency of occurrence of CASP after cervical disc surgery varies between 16\%-26\% [1-9]. Long-term follow-up in a group of patients reveals radiographic evidence (X-ray

\footnotetext{
Received Feb 13, 2015; Revised Apr 13, 2015; Accepted Apr 13, 2015

Corresponding author: Srinivasan Sreeramulu Uddanapalli

Department of Neurosurgery, MIOT Hospitals, 4/112 Mount Poonamalle Road,

Manapakkam, Chennai-600089, Tamil Nadu, India

Tel: +91-9841294495, Fax: +91-4422324570, E-mail: ussrini@vsnl.net
} 
or magnetic resonance imaging [MRI]) of involvement in the form of degenerative changes in the adjacent intervertebral disc space, either above or below the previouslyoperated level, which is termed radiological adjacent segment pathology (RASP). The frequency of RASP varies between $50 \%$ and $92 \%$ [3-5]. A high incidence of CASP was been widely reported [6-14].

The etiology of CASP involves many factors. ASD could be the result of progression of cervical spondylosis to adjacent levels and is not caused by the arthrodesis itself [3]. ASD after interbody fusion could also be multi-factorial [3]. Various changes that occur in the adjacent functional segmental unit like disc degeneration, instability, spinal stenosis, facet degeneration, and deformity have been grouped as ASD. The true incidence and clinical impact of these degenerative changes at the adjacent segment is unclear in the absence of a universally accepted classification system [15]. Seven classification systems including degeneration of adjacent segment exist; but, a systematic review revealed no formal classification system for either cervical or thoracolumbar adjacent segment disorders [15].

Here we present a new classification scheme for CASP with an appropriate line of management, which can be effectively used by surgeons in day-to-day case management. A critical look into our surgical cases revealed CASP can occur in non-operated cervical disc cases; this also has not been studied in depth.

\section{Materials and Methods}

From April 2003 to April 2011, 320 patients with clinically symptomatic cervical disc disease were treated in our hospital. Of these, 509 disc spaces were surgically decompressed and fused through a previously described anterior approach [16]. Operations conducted from May 2011 to May 2014 were not taken into consideration for analysis, since the follow up period would be less than 3 years. Anterior microdiscectomy and fusion using tricortical iliac crest bone graft without plating was performed in 308 patients. Nine patients underwent additional plating with anterior cervical spine-locking plate since they had associated cervical instability at that level. In three other patients, anterior corpectomy and fusion with anterior cervical plating was performed, since they had large sequestrated disc behind the body of the vertebra.

Four cases were lost for follow-up within a month after surgery and were excluded from the study, resulting in a total number of 316 patients. Slippage of the bone graft after one month due to partial anterior migration was observed in three patients. Two of these cases were reoperated on and the graft reimpacted. In addition, anterior cervical plating was performed. The third case refused surgery and was treated with a hard cervical collar for a further 3 months with satisfactory bone fusion.

Clinical evaluation and X-rays of the cervical spine (lateral view) were also carried out periodically, at the end of 1 month in 316 cases (98.7\%), at 6 months in 272 cases (85\%), 1 year in 243 cases ( $75.9 \%)$, and 2 years in 220 cases (68.7\%). Follow-up ranged from a minimum of 3 years to a maximum of 11 years. Since all these patients were operated on by the senior surgeon in a single hospital they could be followed-up for long time, since complete computerized medical records were available.

Patients ( 237 males, 83 females) ranged in age from 28 to 75 years. In all these patients, a detailed evaluation was carried out, involving X-ray and MRI of the cervical spine. Cases where MRI demonstrated a cervical disc disease only were included in the study. Patients with segmental cervical ossified posterior longitudinal ligament and traumatic cervical disc prolapse were excluded. The clinical criteria for surgical intervention in patients presenting with cervical disc disease were definite neurological deficit of either radiculopathy or myelopathy; intolerable radicular pain of more than 6 weeks duration without neurological deficit and no improvement with conservative treatment; or repeated episodes of neck pain of more than 6 months duration with neck stiffness and restriction of neck movements that did not respond to conservative treatment. Radiological criteria were indentation of the thecal sac opposite to the disc space as revealed by MRI of the cervical spine with either definite compression of the exiting nerve root with obliteration of the anterior subarachnoid space (Fig. 1) [3,16,17], anteroposterior spinal cord diameter $<8 \mathrm{~mm}$ as measured by MRI (Fig. 1 ), or hyperintense signal within the spinal cord suggestive of myelomalacia changes present just opposite to the disc space or compressed area (Fig. 1) [17]. Identical MRI criteria were used in the postoperative MRI scan of the cervical spine to define the adjacent segment pathology in the disc space involved following the first surgery. All the MRI scans were independently reviewed by a radiologist. Criteria for surgery in cases with ASD included the aforementioned clinical and MRI criteria. Minimum of 
one clinical criterion and one MRI criterion needed to be present before surgery was advised. X-ray criteria of degeneration in the adjacent disc space of narrowed intervertebral disc space or development of anterior or posterior osteophytes were not considered as symptomatic CASP in patients. In this study only those patients who satisfied the aforementioned MRI criteria in the postoperative scan were considered, with the MRI information correlated with their clinical status. Only symptomatic cases who developed CASP following the first cervical surgery were the focus of this study.

From May 2011 to May 2014, 220 patients responded to

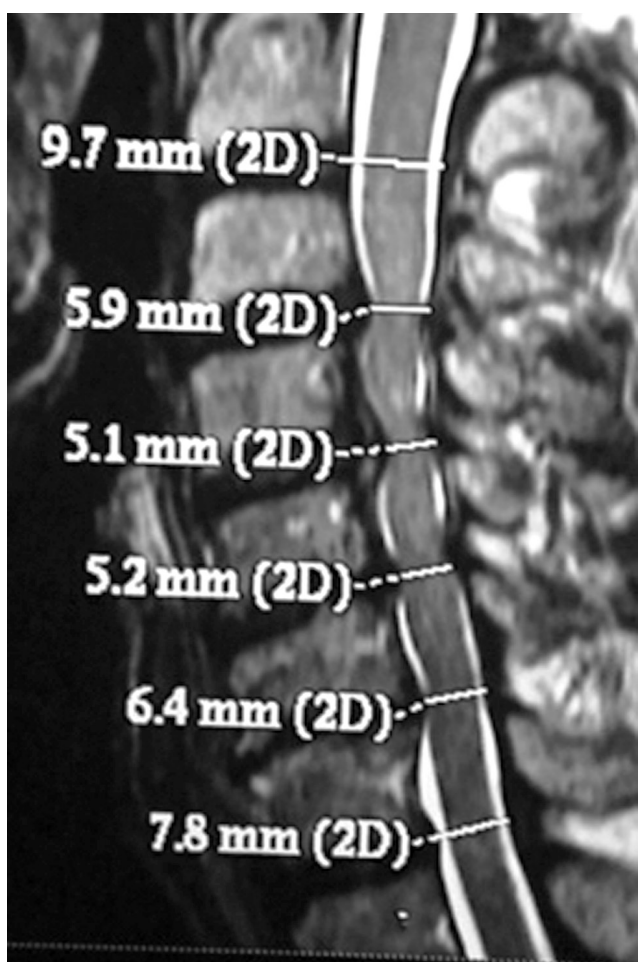

Fig. 1. Magnetic resonance imaging sagittal section showing the criteria followed for subjecting the patient for surgery. our a standard post surgical cervical spine feedback questionnaire and came for a follow-up. Among this group, 165 patients had a postoperative MRI scan of the cervical spine. Preoperative MRI scan of all 165 patients was compared to the postoperative scan from the same patient. The level of the disc space in question was critically evaluated using the MRI criteria. Only those cases which strictly adhered to the clinical and MRI criteria were considered as symptomatic CASP and were subjected to surgical intervention following failure of conservative treatment.

\section{Results}

Of the 316 patients, 6 (1.9\%) developed symptomatic CASP requiring resurgery. There were eight cases of MRI proven CASP with axial neck pain only without neurological deficit (Table 1). This group of patients was contraindicated for surgery since they had only tolerable axial neck pain. They are being periodically followed up. Thirteen asymptomatic patients who were followed-up had MRI evidence of early stage of RASP (Table 1). CASP following surgery developed in 27 of the 316 patients (8.5\%). Follow-up MRI scans were done in 165 patients; of these, $27(16.3 \%)$ revealed variable degree of indentation of the thecal sac in adjacent disc spaces. Of the 27, $6(3.6 \%)$ required surgery. Four of the 316 cases (1.3\%) that did not receive surgery developed symptomatic CASP, due either to congenital fusion or degenerative fusion of vertebra requiring surgery. Among the 316 patients, 130 patients are being followed up for more than 6 years.

CASP occurred in some cases where surgery was not done, involving congenital or degeneration fusion. This led us to a novel classification of CASP as primary or secondary CASP. Primary (A) comprises A1 (congenital fusion of vertebra, such as Klippel Feil vertebra) (Fig. 2) and A2

Table 1. Showing the various subheadings under which adjacent segment pathology cases can manifest

\begin{tabular}{llc} 
No. & Type of adjacent segment pathology in cervical disc disease & No. of cases under each category (\%) \\
\hline 1 & CASP requiring resurgery in entire series of 316 cases. & $6(1.89)$ \\
\hline 2 & CASP requiring resurgery in 165 cases where MRI was taken. & $6(3.63)$ \\
\hline 3 & RASP who are asymptomatic in 165 cases. & $8(7.88)$ \\
\hline 4 & CASP with axial pain only without neurological deficit in 165 cases. & 2 \\
\hline 5 & Congenital cervical fusion cases who developed CASP requiring surgery. & 2 \\
\hline 6 & Degenerative cervical fusion cases who developed CASP requiring surgery. & 85.89 \\
\hline
\end{tabular}

CASP, clinical adjacent segment pathology; MRI, magnetic resonance imaging; RASP, radiological adjacent segment pathology. 
(degenerative fusion of vertebra) (Fig. 3). Secondary (B) CASP is when adjacent degeneration occurs after cervical disc surgery. Subdivisions include B1 (RASP in asymp-

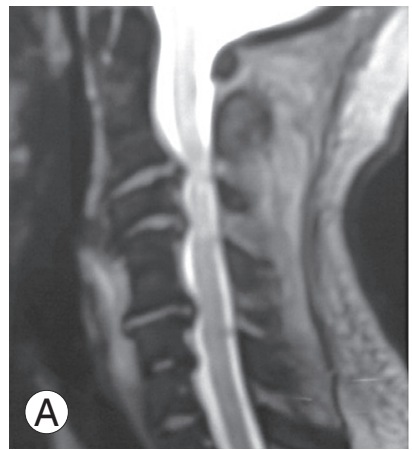

MRI showing C2-C3, C3-C4 cervical disc prolapse with cord compression with C4-C5 fusion of vertebra

Fig. 2. Congenital fusion of vertebra. (A) Magnetic resonance imaging showing clinical adjacent segment pathology in congenital fusion of vertebra. (B) X-ray lateral view of the same case is shown.

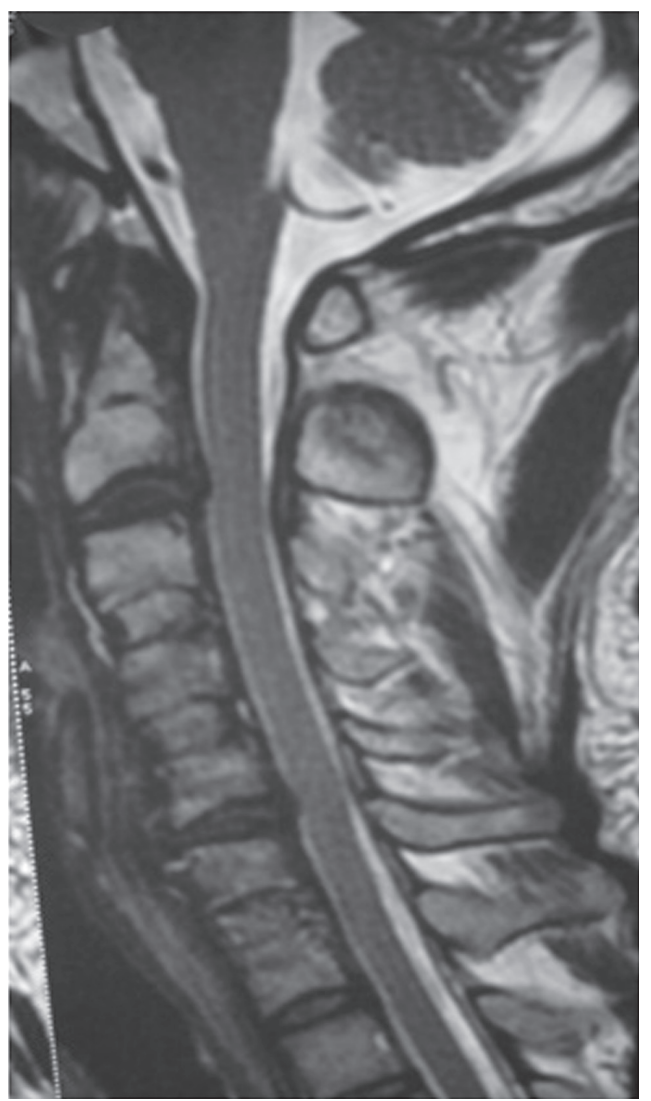

Fig. 4. An asymptomatic patient who had underwent two level fusion at C3-C4, and C4-C5. A sagittal section magnetic resonance imaging scan showed minimal indentation at C2-C3, and C6-C7 levels, suggestive of radiological adjacent segment pathology. tomatic patients) (Fig. 4), B2 (CASP in patients with axial neck pain only) (Fig. 5), and B3 (CASP with myeloradiculopathy) (Figs. 6, 7). B3 I subdivided into B3a (single level CASP) (Fig. 6) and B3b (multiple level CASP) (Fig. 7).

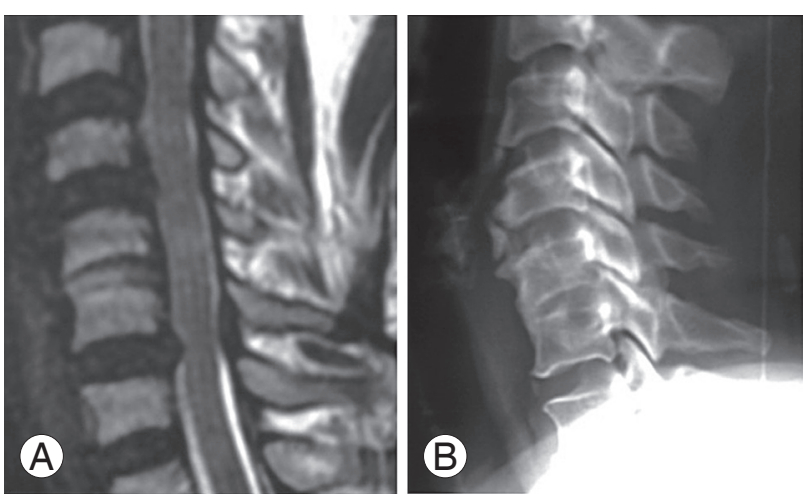

Fig. 3. (A) Magnetic resonance imaging apparent clinical adjacent segment pathology at C6-C7 disc space in a case of degenerative fusion of C5-C6 vertebra. (B) X-ray lateral view of the same case is shown.

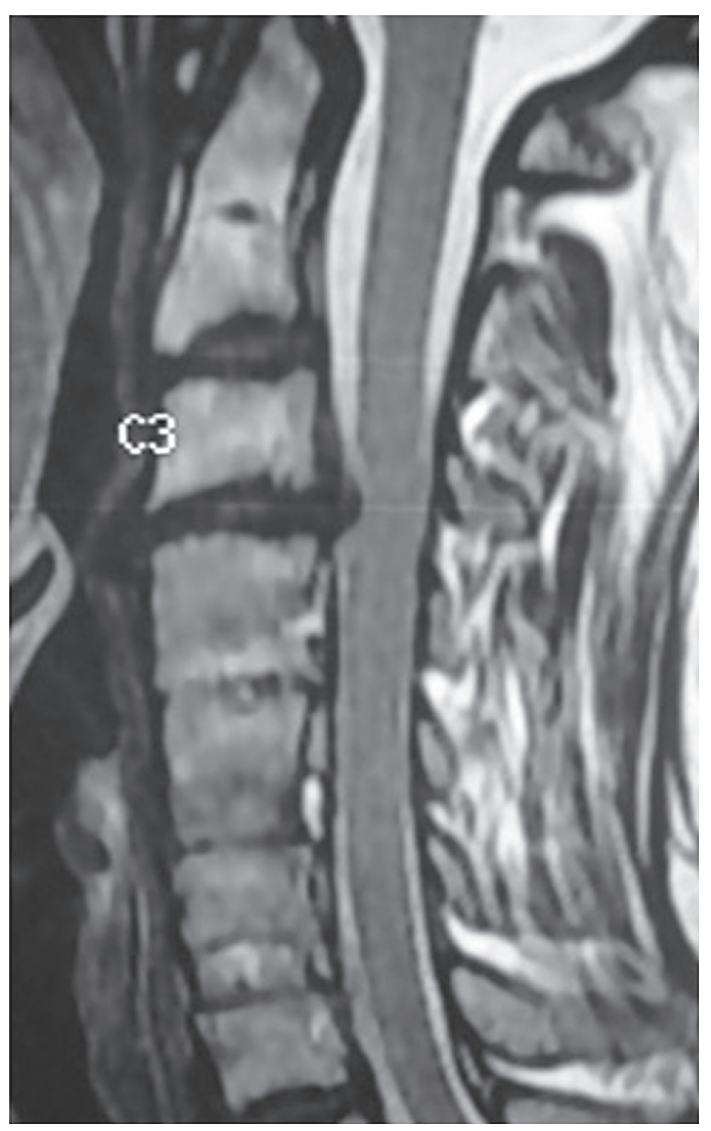

Fig. 5. Sagittal section magnetic resonance imaging scan showing indentation at C3-C4 level, suggestive of adjacent segment pathology, in a case featuring three level fusions at C4-C7 and only axial neck pain. 

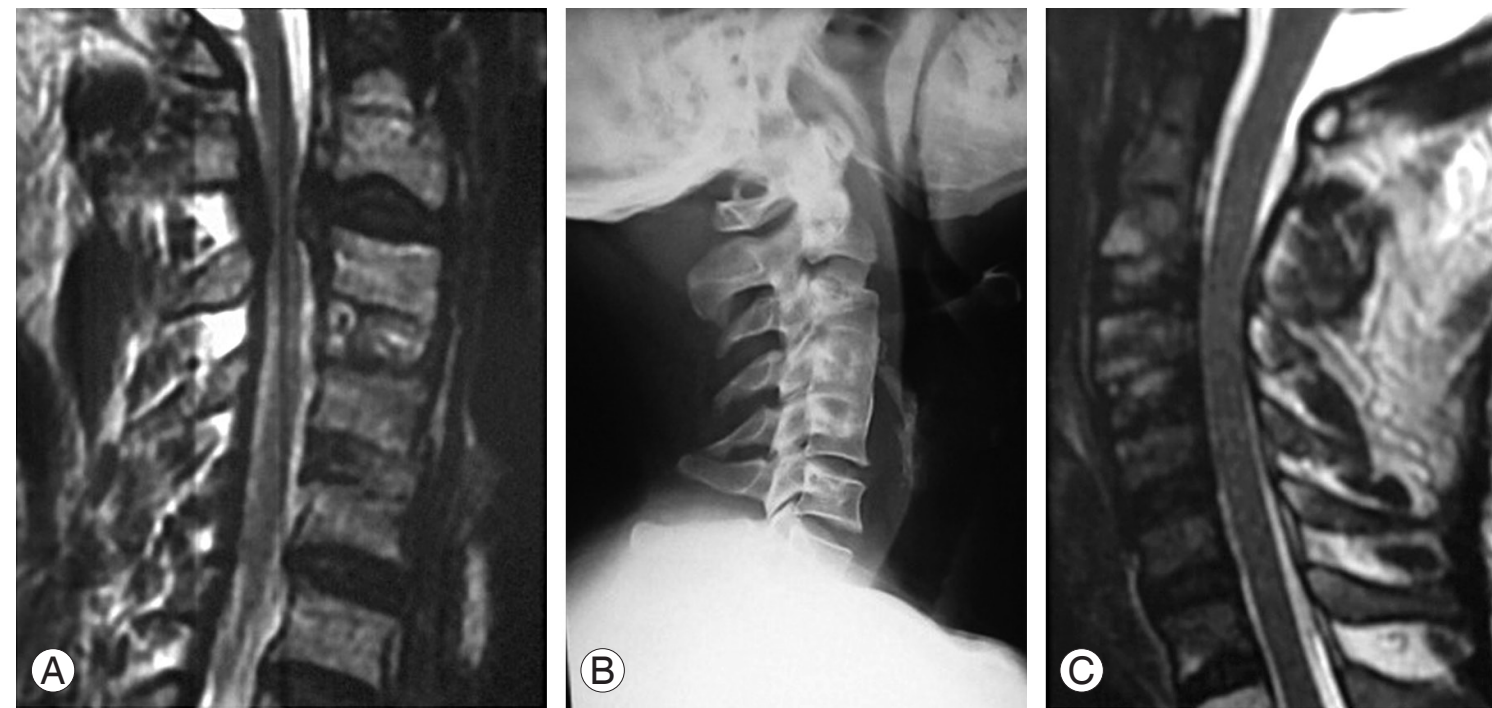

Fig. 6. Postoperative clinical adjacent segment pathology at the C2-C3 level, in a case operated for cervical disc prolapse at C3C4, and C4-C5. (A) Preoperative magnetic resonance imaging (MRI) cervical spine showing C2-C3 cord compression opposite to the disc space (note: bone grafts seen at C3-C4, C4-C5 the site of previous disc surgery). (B) Postoperative X-ray showing a bone graft at C2-C3 (note: completely fused bone grafts at C3-C4, C4-C5). (C) Postoperative MRI scan showing no compression at C2C3 the area of resent surgery).
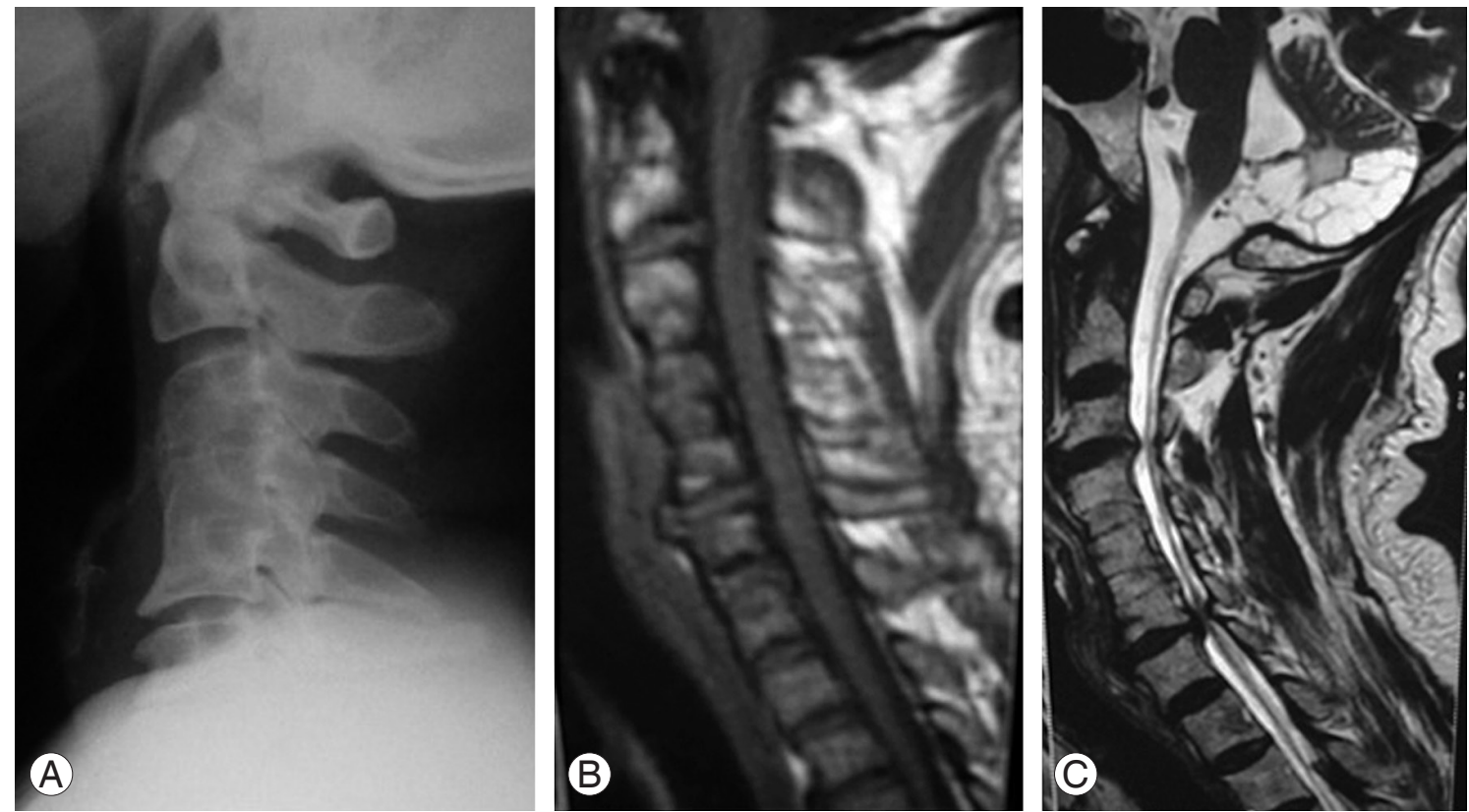

Fig. 7. Magnetic resonance imaging (MRI) apparent clinical adjacent segment pathology at multiple levels. (A) X-ray showing excellent fusion of $\mathrm{C} 3-\mathrm{C} 4, \mathrm{C} 4-\mathrm{C} 5$ bone grafts at the site of previous disc surgery. (B) MRI showing adjacent segment degeneration (ASD) at C2-C3, C5-C6 multiple levels. (C) MRI showing ASD at C3-C4, C6-C7 at multiple levels (note: completely fused bone grafts at $\mathrm{C} 4-\mathrm{C} 5, \mathrm{C} 5-\mathrm{C} 6$ the area of previous disc surgery).

\section{Discussion}

CASP is the development of new degenerative disc disease at an immediately contiguous level, either cephalad and/ or caudal to the previous arthrodesis $[3,15]$. Indentations of the dura matter on myelogram and disc protrusion on MRI are significantly more frequent among those who developed cervical CASP than those who remain disease 
free $[3,4]$. This pattern was also observed presently. However, the incidence of CASP was $\mathrm{c}$ high. In our study with a maximum follow-up of up to 11 years, (which included 130 patients with a follow-up of 6 years) the frequency of resurgery was $1.9 \%(6 / 316)$. If follow-up MRI scan group only was considered, 3.6\% (6/165) required resurgery (Fig. 6) because of symptomatic CASP out of 27 cases whose MRI scans revealed variable degree of indentation in adjacent disc spaces, which did not warrant any surgical intervention (Figs. 4, 5). This low incidence appears to be at variance with the incidence reported elsewhere [3$5,18,19]$. Kienapfel et al. [6] reported that only significant myelocompression, and not just adjacent instability, was associated with statistically significant low quality of life scores. We agree.

Further critical analysis of the 320 cases revealed four cases of disc prolapse in those with congenital fusion (Fig. 2) or degenerative fusion (Fig. 3) of vertebra, which required surgery. Even those with congenital fusion of vertebra present since birth developed CASP after 35-50 years. In those who had spontaneous degenerative fusion, CASP occurred after the age of 50 years. We cannot conclude that there is a very high risk of developing ASD following fusion surgery.

In the A1 classification (primary: congenital fusion) there were two cases of congenital fusion of cervical vertebra who presented with progressive myeloradiculopathy requiring surgery. The first case had congenital fusion of C4-C5 vertebra. MRI revealed severe compression of the cervical spinal cord in adjacent disc level C2-C3, C3-C4 (Fig. 2). C2-C3, and C3-C4 anterior microdiscectomy and fusion was performed. The second case had C4-C5 congenital fusion with cord compression at $\mathrm{C} 3-\mathrm{C} 4$ due to well developed posterior osteophyte (Fig. 8). A C3$\mathrm{C} 4$ anterior microdiscectomy and fusion was performed using an iliac crest bone graft. Both patients improved
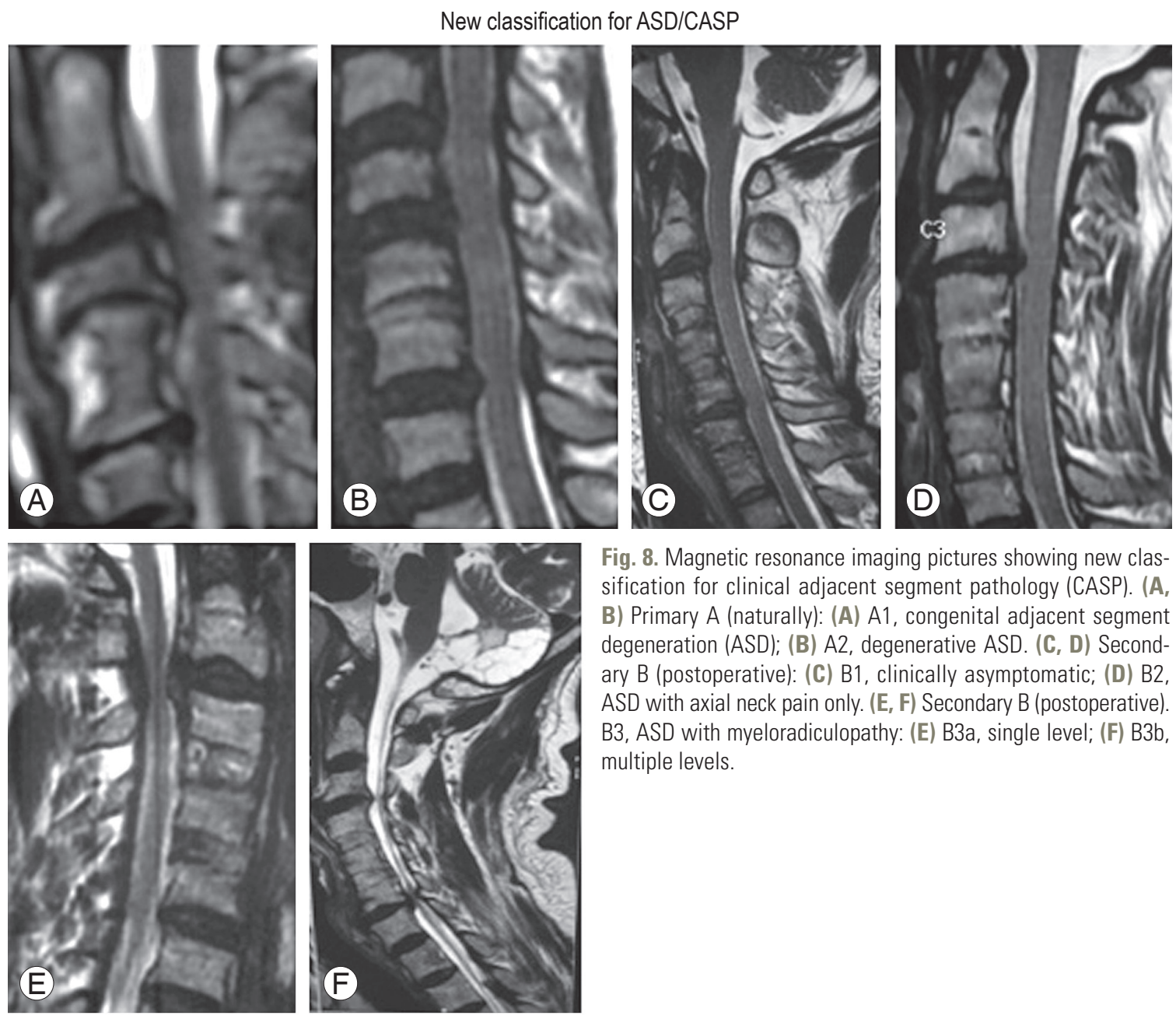

Fig. 8. Magnetic resonance imaging pictures showing new classification for clinical adjacent segment pathology (CASP). (A, B) Primary $A$ (naturally): (A) A1, congenital adjacent segment degeneration (ASD); (B) A2, degenerative ASD. (C, D) Secondary B (postoperative): (C) B1, clinically asymptomatic; (D) B2, ASD with axial neck pain only. (E, F) Secondary B (postoperative). B3, ASD with myeloradiculopathy: (E) B3a, single level; (F) B3b, multiple levels. 
neurologically. Group A2 (primary: degenerative fusion) also comprised two cases. Degenerative fusion was noted in both cases at $\mathrm{C} 5-\mathrm{C} 6$ and cord compression at adjacent level of C6-C7 (Fig. 3). Both patients presented with progressive myeloradiculopathy and were operated on.

RASP was presently evident in 13 patients (Fig. 4) as reported in the literature [9], but they are asymptomatic (Table 1). They were categorized as B1 (RASP in asymptomatic patients). These patients would not have been part of the regular follow-up because of their asymptomatic status. But in case they are seen by the treating physician, patients should be managed as suggested for B2 group. There were eight B2 patients (CASP with axial neck pain only; Table 1) who had axial pain only without any clinical signs of myeloradiculopathy. Their MRI scan showed minimal indentation (Fig. 5) opposite to the disc space without any signal changes within the spinal cord. They are being treated conservatively with intensive neck muscle strengthening exercise program. These groups of patients are being periodically followed up once in 3 months.

The B3 group (CASP with myeloradiculopathy) comprised six cases who required resurgery (Fig. 6). Among these, four cases had single level CASP (B3a) (Fig. 6) and two had multiple level CASP (B3b) (Fig. 7). All presented with neck pain and progressive myeloradiculopathy. One patient with multiple level CASP at C3-C4, C6-C7 (Fig. 7) had Nurick's grade 5 [20] when presenting for surgery. Following surgery there was only minimal clinical improvement with reduction in the frequency of flexor spasms, but the patient remained wheel chair bound. Other patients who presented in grade 2 or 3 Nurick's grade showed marked improvement in their neurological status after surgery. In all these cases anterior cervical microdiscectomy with excision of the compressing posterior osteophytes was performed. In the B3b subgroup, where multiple levels of CASP are present, we fused at the above segment and did a simple decompression at the below segment without fusion.

Based upon the classification which we have proposed, patients in group A1 (Fig. 2) or A2 (Fig. 3) who are symptomatic and have definite evidence of myeloradiculopathy, and B3 showing compression at single (Fig. 6) or multiple (Fig. 7) level require surgical intervention. In the B3b subgroup where multiple levels of CASP are present, we prefer to fuse at the above segment and do a simple decompression at the below segment without fusion so that minimal range of neck mobility is preserved for the patient. In the B2 group, patients who present only with axial pain even though their MRI scan shows minimal indentation (Fig. 5) need conservative treatment, since surgery for axial pain only is not of much benefit to the patient [21-24]. They have to be put on intensive neck muscle strengthening exercises, which likely will prevent them from developing CASP in the future. They need to be monitored clinically once every 3 months and radiologically using MRI scans once annually. At the early evidence of this group of patients developing clinically symptomatic myeloradiculopathy, MRI scan has to be repeated. If reoperation is needed, surgery should be done before the development of hyperintense signal within the MRI scan to prevent neurologic deterioration.

The type of surgery performed in those who develop CASP is an issue. Many patients who develop CASP were subjected to posterior foraminotomy in single level disc disease $[9,12,14]$. Proponents of the use of the artificial disc strongly advocate its' use to prevent CASP [3,5$7,18,19]$. Whether total disc replacement (TDR) would be useful in this group who require resurgery is uncertain, since even the occurrence of CASP in those who underwent TDR as the first surgery [10-12,14,18,19,25]. There is reportedly no statistically significant difference in the incidence of development of CASP between TDR and anterior cervical discectomy and fusion (ACDF) $[1,2,10$ $12,14,25]$. This observation supports our decision to perform ACDF even in those cases who developed CASP following previous surgery and in naturally occurring congenital and degenerative fusion cases who presented with myelopathy. In CASP cases who present with radiculopathy, posterior foraminotomy may be the procedure of choice. In those cases who still present with myelopathy, ACDF is the best option $[13,26]$, since the anterior cord compression is relieved (Fig. 6), which would help the patient in recovery.

A critical relook into the progression of the disease process in these cases who developed CASP revealed that in those following surgery the interval period may vary between 3-10 years, as in our series. Still, within 10 years there is a chance of occurrence of CASP. Presently, when congenital or degenerative cases were included, symptomatic CASP occurred after more than 40 years of life. In congenital cases fusion is present since birth. Still, the adjacent disc space may not undergo degeneration for a long time. Also, the overall prevalence or incidence is 

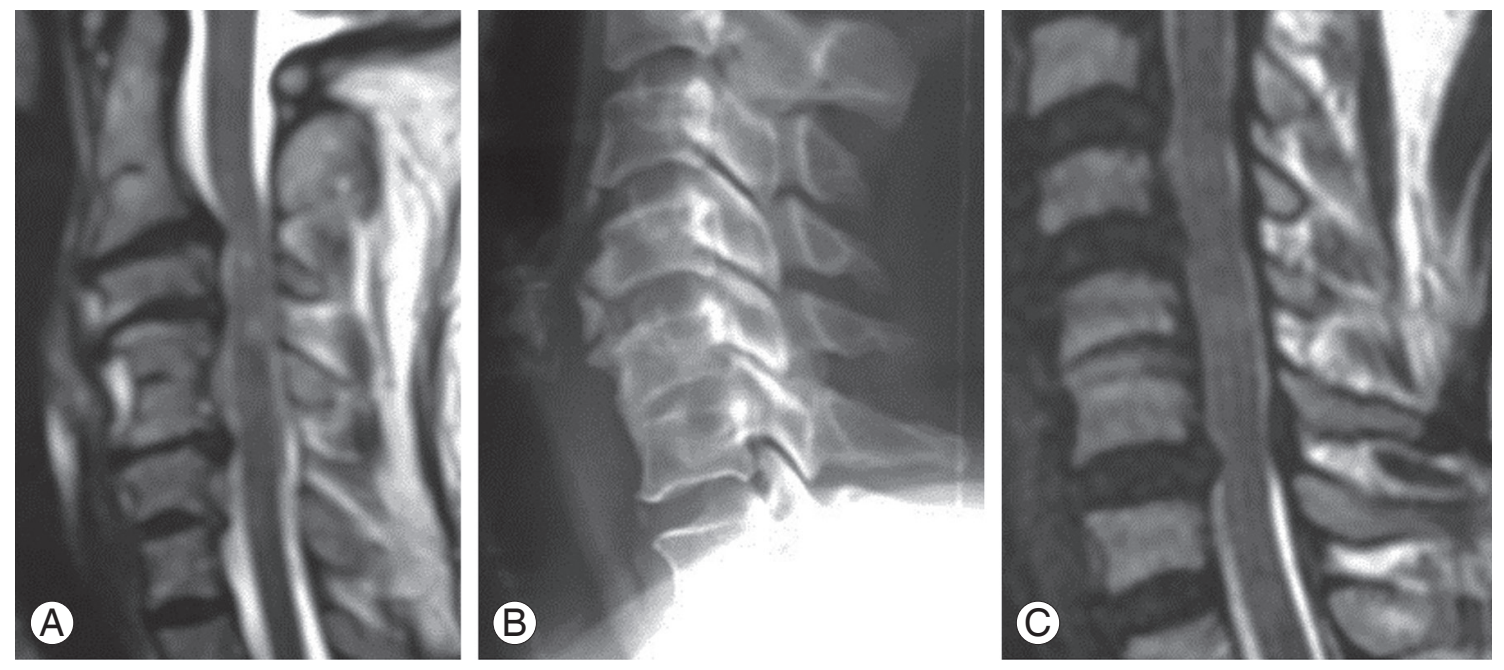

Fig. 9. Radiologically apparent maintenance of the disc space in congenital and degenerative fusions. (A) Magnetic resonance imaging (MRI) in congenital fusion of $\mathrm{C} 4-\mathrm{C} 5$ showing the maintenance of the disc space. (B) X-ray and (C) MRI in degenerative fusion showing the maintenance of the C5-C6 disc space above the C6-C7 cord compression.

markedly less when other non-symptomatic congenital fusions are also considered. Degenerative fusion occurs only over 50 years of age, and the prevalence or incidence is also very low.

With these factors in mind, we reexamined the X-ray and MRI scans of the patients with congenital and degenerative fusions. Only partial fusion of a part of the vertebra was apparent, whether it was between the bodies, where disc space was visible (Fig. 9), or at other parts of the vertebra. These factors may have prevented accelerated degeneration of the adjacent segment by maintaining a degree of mobility in the segment where either congenital fusion or degenerative fusion has occurred. A deeper examination of this aspect may give a clue to future longterm prevention of CASP, even in these minority cases of surgically treated patients. It is important to classify and identify since the etiology for CASP in both these groups differs. This has led us to logically offer a sub classification (Fig. 8), which is presented here.

\section{Conclusions}

Symptomatic CASP requiring resurgery is infrequent. CASP can occur in patients with congenital and degenerative fusion of the cervical spine. A new classification for CASP along with the treatment strategy is proposed. Regarding the management of the patients falling within these groups, we suggest that surgery be reserved only for the primary CASP group and B3 CASP with myelora- diculopathy, with other patients requiring only periodic observation.

\section{Conflict of Interest}

No potential conflict of interest relevant to this article was reported.

\section{Acknowledgments}

We thank Prof. Dr. P.V.A. Mohandas, managing director, MIOT Hospitals, Chennai, India for permitting me to do the above work. Dr. Madan senior consultant radiologist for independently reviewing the MRI scans of the patients.

\section{References}

1. Lawrence BD, Hilibrand AS, Brodt ED, Dettori JR, Brodke DS. Predicting the risk of adjacent segment pathology in the cervical spine: a systematic review. Spine (Phila Pa 1976) 2012;37(22 Suppl):S52-64.

2. Harrod CC, Hilibrand AS, Fischer DJ, Skelly AC. Adjacent segment pathology following cervical motionsparing procedures or devices compared with fusion surgery: a systematic review. Spine (Phila Pa 1976) 2012;37(22 Suppl):S96-112.

3. Hilibrand AS, Carlson GD, Palumbo MA, Jones PK, Bohlman HH. Radiculopathy and myelopathy at seg- 
ments adjacent to the site of a previous anterior cervical arthrodesis. J Bone Joint Surg Am 1999;81:51928.

4. Ishihara H, Kanamori M, Kawaguchi Y, Nakamura $\mathrm{H}$, Kimura T. Adjacent segment disease after anterior cervical interbody fusion. Spine J 2004;4:624-8.

5. Janssen ME, Marchesi DG. Adjacent segment disease as a result of fusion in the cervical spine (C-ASD): prognostic factors. Evid Based Spine Surg 2008;4:5566.

6. Kienapfel H, Koller M, Hinder D, et al. Integrated outcome assessment after anterior cervical discectomy and fusion: myelocompression but not adjacent instability affect patient-reported quality of life and cervical spine symptoms. Spine (Phila Pa 1976) 2004; 29:2501-9.

7. Bartolomei JC, Theodore N, Sonntag VK. Adjacent level degeneration after anterior cervical fusion: a clinical review. Neurosurg Clin N Am 2005;16:57587.

8. An C, Guo J, Yuan Q. Clinical observation to adjacent-segment disease after anterior cervical discectomy and fusion. Zhongguo Xiu Fu Chong Jian Wai Ke Za Zhi 2008;22:390-3.

9. Xu R, Bydon M, Macki M, et al. Adjacent segment disease after anterior cervical discectomy and fusion: clinical outcomes after first repeat surgery versus second repeat surgery. Spine (Phila Pa 1976) 2014; 39:120-6.

10. Maldonado CV, Paz RD, Martin CB. Adjacent-level degeneration after cervical disc arthroplasty versus fusion. Eur Spine J 2011;20 Suppl 3:403-7.

11. Coric D, Nunley PD, Guyer RD, et al. Prospective, randomized, multicenter study of cervical arthroplasty: 269 patients from the Kineflex $\mid \mathrm{C}$ artificial disc investigational device exemption study with a minimum 2-year follow-up: clinical article. J Neurosurg Spine 2011;15:348-58.

12. Singh K, Phillips FM, Park DK, Pelton MA, An HS, Goldberg EJ. Factors affecting reoperations after anterior cervical discectomy and fusion within and outside of a Federal Drug Administration investigational device exemption cervical disc replacement trial. Spine J 2012;12:372-8.

13. Srinivasan US, Mahesha K, Senthilkumar G. Symptomatic adjacent segment degeneration in cervical disc disease requiring surgical intervention: single author experience. J Spinal Surg 2012;3:725-8.

14. Coric D, Kim PK, Clemente JD, Boltes MO, Nussbaum M, James S. Prospective randomized study of cervical arthroplasty and anterior cervical discectomy and fusion with long-term follow-up: results in 74 patients from a single site. J Neurosurg Spine 2013;18:36-42.

15. Kraemer P, Fehlings MG, Hashimoto R, et al. A systematic review of definitions and classification systems of adjacent segment pathology. Spine (Phila Pa 1976) 2012;37(22 Suppl):S31-9.

16. Robinson RA, Smith GW. Anterolateral cervical disc removal and interbody fusion for cervical disc syndrome. Bull Johns Hopkins Hosp 1955:96:223-4.

17. Czervionke LF, Haughton VM. Degenerative disease of the spine. In: Atlas SC, editor. Magnetic resonance imaging of the brain and spine. 3rd ed. Philadelphia: Lippincot William \& Wilkins; 2002. p. 1633-714.

18. Murrey D, Janssen M, Delamarter R, et al. Results of the prospective, randomized, controlled multicenter Food and Drug Administration investigational device exemption study of the ProDisc-C total disc replacement versus anterior discectomy and fusion for the treatment of 1-level symptomatic cervical disc disease. Spine J 2009;9:275-86.

19. Zigler JE, Delamarter R, Murrey D, Spivak J, Janssen M. ProDisc-C and anterior cervical discectomy and fusion as surgical treatment for single-level cervical symptomatic degenerative disc disease: five-year results of a Food and Drug Administration study. Spine (Phila Pa 1976) 2013;38:203-9.

20. Nurick $S$. The pathogenesis of the spinal cord disorder associated with cervical spondylosis. Brain 1972;95: 87-100.

21. Eck JC, Humphreys SC, Hodges SD, Levi P. A comparison of outcomes of anterior cervical discectomy and fusion in patients with and without radicular symptoms. J Surg Orthop Adv 2006;15:24-6.

22. Garvey TA, Transfeldt EE, Malcolm JR, Kos P. Outcome of anterior cervical discectomy and fusion as perceived by patients treated for dominant axialmechanical cervical spine pain. Spine (Phila Pa 1976) 2002;27:1887-95.

23. Palit M, Schofferman J, Goldthwaite N, et al. Anterior discectomy and fusion for the management of neck pain. Spine (Phila Pa 1976) 1999;24:2224-8.

24. Riew KD, Ecker E, Dettori JR. Anterior cervical dis- 
cectomy and fusion for the management of axial neck pain in the absence of radiculopathy or myelopathy. Evid Based Spine Care J 2010;1:45-50.

25. Anderson PA, Sasso RC, Hipp J, Norvell DC, Raich A, Hashimoto R. Kinematics of the cervical adjacent segments after disc arthroplasty compared with anterior discectomy and fusion: a systematic review and meta-analysis. Spine (Phila Pa 1976) 2012;37(22 Suppl):S85-95.

26. Arnold P, Boswell S, McMahon J. Threaded interbody fusion cage for adjacent segment degenerative disease after previous anterior cervical fusion. Surg Neurol 2008;70:390-7. 